\title{
Regularization and Convergence for Singular Perturbations
}

\author{
H. Neidhardt ${ }^{1}$ and V.A. Zagrebnov ${ }^{2, \star}$ \\ 1 Technische Universität Berlin, Fachbereich 3 Mathematik, MA 7-2, Strasse des 17. Juni 136, \\ W-1000 Berlin 12, FRG \\ 2 Instituut voor Theoretische Fysica, Katholieke Universiteit Leuven, Celestijnenlaan 200 D, B-3001 \\ Leuven, Belgium
}

Received April 16, 1991; in revised form May 1, 1992

\begin{abstract}
We present an abstract result on removing regularization for singular perturbations in the operator theory. Our main result concerns singular perturbations which are not (formally) semibounded from below.
\end{abstract}

\section{Introduction}

For very singular and nonpositive potentials $-V, V \geq 0$, in quantum mechanics it may happen that the Schrödinger operator $H=-\Delta-V$ makes no sense, i.e., it is not essentially self-adjoint on $\mathscr{D}(-\Delta) \cap \mathscr{D}(V)$. Further, if we formally define the Schrödinger operator on $\mathscr{D}(-\Delta)$, then $H$ is not semibounded from below. However, one can choose a regularizing sequence $\left\{V_{n}\right\}_{n=1}^{\infty}$ of bounded potentials such that $V_{n} f \rightarrow V f$ as $n \rightarrow \infty$ for certain elements $f \in \mathscr{D}(V)$ and the corresponding sequence $\left\{H_{n}\right\}_{n=1}^{\infty}$ of well-defined Schrödinger operators, i.e. $H_{n}=-\Delta-V_{n}$, such that the limit s- $\lim _{n \rightarrow \infty}\left(H_{n}-z\right)^{-1}, \operatorname{Im}(z) \neq 0$, exists and defines a self-adjoint operator which can be understood as a regularized Schrödinger operator for the problem $H=-\Delta-V$ $[4,5,7,8,13]$. It is interesting to note that the regularizing sequence $\left\{V_{n}\right\}_{n=1}^{\infty}$ itself chooses the "right" regularized Schrödinger operator or, in other words, that the singular perturbation itself forces the "right" operator.

The present paper has the aim to clarify this phenomenon on an abstract operatortheoretical level. Thus, the paper is closely related to [8]. The main result of [8] (Theorem 1) say that if

(i) the regularizing sequence $\left\{V_{n}\right\}_{n=1}^{\infty}, \mathscr{D}\left(V_{n}\right) \supset \mathscr{D}(V)$, satisfies $V_{n} \leq V$,

(ii) there exists a dense subset $\mathscr{D} \subseteq \mathscr{D}(A) \cap \mathscr{D}(V)$ such that

$$
\|V f\| \leq a\|A f\|+b\|f\|, \quad a<1, \quad b<+\infty, \quad f \in \mathscr{D},
$$

\footnotetext{
* On leave of absence from Laboratory of Theoretical Physics, Joint Institute for Nuclear Research, Dubna 141980, CIS (Russia)
} 


$$
\begin{gathered}
\left\|\left(V-V_{n}\right) f\right\| \leq a_{n}\|A f\|+b_{n}\|f\|, \quad \lim _{n \rightarrow \infty} a_{n}=\lim _{n \rightarrow \infty} b_{n}=0 \quad f \in \mathscr{D}, \\
(A-V) \mid \mathscr{D} \text { has finite deficiency indices }(m, m) .
\end{gathered}
$$

(iii) there exists $\left\{c_{n}\right\}_{n=1}^{\infty}, \lim _{n \rightarrow \infty} c_{n}=+\infty$, such that the spectrum of $H_{n}=A-V_{n}$ contained in $\left(-\infty,-c_{n}\right)$ consists of at least $m$ eigenvalues (counting multiplicities), then the sequence $\left\{H_{n}\right\}_{n=1}^{\infty}$ converges in the norm resolvent sense to the Friedrichs extension $H_{F}$ of $(A-V) \mid \mathscr{D}$ as $n \rightarrow \infty$. In addition, it is shown that the operators $H_{n} \mid \mathscr{D}$ are uniformly bounded from below. ${ }^{1}$

In the following we show that these conditions can be relaxed. For example, it is not necessary that the involved self-adjoint operators are self-adjoint extensions of a common symmetric operator with finite deficiency indices (condition (ii) (3)). However, provided that this holds, our results can be improved essentially, as can be seen in the third section. Moreover, it becomes clear that the notion of an admissible regularizing sequence is crucial for the problem. Further, it turns out that it is enough to consider closed symmetric forms.

The problem of approximation and convergence was studied on the abstract level in $[3,6,9,10]$. These results were applied to singular perturbations semibounded from below (see e.g. $[11,12])$ and to the strong singular case with hard-core (see e.g. [14] and the recent book [1] and references therein).

\section{Regularization and Convergence}

Let $\nu \geq 0$ be a closed symmetric form defined on a complex separable Hilbert space $\mathfrak{h}$ and let $A$ be its associated self-adjoint operator, i.e. $\nu(f, g)=(\sqrt{A} f, \sqrt{A} g)$, $f, g \in \mathscr{D}(\sqrt{A}) \equiv \operatorname{dom}(\nu)$. Let $\mathscr{D}$ be a dense subset of $\operatorname{dom}(\nu)$. Since $\nu$ is a closed symmetric form, the form $\nu_{0}$ given by

$$
\nu_{0}(f, g)=\nu(f, g), \quad f, g \in \mathscr{D}
$$

is closable. By $\hat{\nu}$ we denote the closure of $\nu_{0}$. Obviously, we have $\hat{\nu} \geq 0$, $\operatorname{dom}(\hat{\nu}) \subseteq \operatorname{dom}(\nu)$, and

$$
\nu \mid \operatorname{dom}(\hat{\nu})=\hat{\nu} .
$$

The associated self-adjoint operator of $\hat{\nu}$ is denoted by $\hat{A}$.

Further, let $\gamma \geq 0$ be a symmetric form such that

$$
\left\{\begin{array}{l}
\mathscr{D} \subseteq \operatorname{dom}(\gamma) \\
\gamma(f, f) \leq a \nu(f, f)+b\|f\|^{2}, f \in \mathscr{D}, 0 \leq a, b .
\end{array}\right.
$$

On account of (2.2) the relation (2.3) can be rewritten as follows:

$$
\gamma_{D}(f, f) \leq a \hat{\nu}(f, f)+b\|f\|^{2}, \quad f \in \mathscr{D}, \quad 0 \leq a, b,
$$

where $\gamma_{D}=\gamma \mid \mathscr{D}$ is the restriction of $\gamma$ to the subset $\mathscr{D}$. But from (2.4) we immediately obtain the estimate $\gamma_{D}(f, f) \leq c\left(\hat{\nu}(f, f)+\|f\|^{2}\right), f \in \mathscr{D}, c=\max \{a, b\}$,

\footnotetext{
1 Notice that in [8] the operators $H_{n} \mid \mathscr{D}(A) \cap \mathscr{D}(V)$ were considered. However, it was proved that $\mathscr{D}(A) \cap \mathscr{D}(V)=\mathscr{D}$ under above conditions
} 
which shows that $\gamma_{D}$ can be continuously extended to elements of dom $(\hat{\nu})$. Denoting the extended form by $\hat{\gamma} \operatorname{dom}(\hat{\gamma})=\operatorname{dom}(\hat{\nu})$, it is not hard to see that $\hat{\gamma}$ is relatively bounded with respect to $\hat{\nu}$, i.e., we have

$$
\hat{\gamma}(f, f) \leq a \hat{\nu}(f, f)+b\|f\|^{2}, \quad f \in \operatorname{dom}(\hat{\nu}), \quad 0 \leq a, b .
$$

We say that the symmetric form $\hat{\gamma}$ is relatively compact with respect to $\hat{\nu}$ if for some $\lambda<0$ the bounded symmetric form $\hat{\beta}(f, g)=\hat{\gamma}\left((\hat{A}-\lambda)^{-1 / 2} f,(\hat{A}-\lambda)^{-1 / 2} g\right)$, $f, g \in \mathfrak{h}$, defines on $\mathfrak{h}$ a compact operator. Notice that if this condition is fulfilled for some $\lambda<0$, then it also holds for all negative $\lambda$.

The KLMN-Theorem [10, Theorem X.17] yields that the perturbed symmetric form $\hat{\mu}_{\kappa}$

$$
\hat{\mu}_{\kappa}(f, g)=\hat{\nu}(f, g)-\frac{1}{\kappa} \hat{\gamma}(f, g), \quad f, g \in \operatorname{dom}(\hat{\mu})=\operatorname{dom}(\hat{\nu})
$$

is closed and bounded from below for $\kappa>a$. We denote the associated self-adjoint operator and the lower bound of $\hat{\mu}_{\kappa}$ by $\hat{H}_{\kappa}$ and $\hat{\lambda}_{\kappa}$, respectively.

Example 2.1. Let $A=-\frac{d^{2}}{d x^{2}}$ be the usually defined Laplace operator on the Hilbert space $\mathfrak{h}=L^{2}\left(\mathbb{R}^{1}\right)$. We set

$$
\nu(f, g)=\int_{-\infty}^{+\infty} f^{\prime}(x) \overline{g^{\prime}(x)} d x
$$

$f, g \in \operatorname{dom}(\nu)=\mathscr{D}(\sqrt{A})=W_{2}^{1}\left(\mathbb{R}^{1}\right)$. Obviously, we have $\nu \geq 0$. Setting $\mathscr{D}=C_{0}^{\infty}\left(\mathbb{R}^{1} \backslash\{0\}\right)$ we can identify the self-adjoint operator $\hat{A}$, roughly speaking, with the Laplace operator which satisfies at zero the boundary condition $f(0)=0$. More precisely, $\hat{A}$ is the Friedrichs extension of the closure $\stackrel{\circ}{A}$ of the restriction $A \mid \mathscr{D}$.

We introduce the quadratic form $\gamma_{\alpha}$ as follows:

$$
\gamma_{\alpha}(f, g)=\frac{1}{4} \int_{-\infty}^{+\infty} \frac{1}{|x|^{\alpha}} f(x) \overline{g(x)} d x, \quad 0 \leq \alpha \leq 2
$$

$f, g \in \operatorname{dom}\left(\gamma_{\alpha}\right)=\mathscr{D}$. Using the corresponding considerations of [6, VI, Sect. 4] we find the estimate

$$
\gamma_{\alpha}(f, f) \leq a_{\alpha} \nu(f, f)+b\left(a_{\alpha}\right)\|f\|^{2}, \quad f \in \mathscr{D}, \quad 0 \leq \alpha \leq 2
$$

where $a_{\alpha}$ can be chosen arbitrary small if $0 \leq \alpha<2$ and $a_{\alpha=2}=1$. The estimate (2.9) proves the estimate (2.3).

Definition 2.2. A sequence $\left\{\gamma_{n}\right\}_{n=1}^{\infty}$ of bounded non-negative symmetric forms on $\mathfrak{h}$ is called a regularizing sequence of $\gamma$ with respect to $\mathscr{D} \subseteq \operatorname{dom}(\gamma)$ if

(i) $0 \leq \gamma_{1} \leq \gamma_{2} \leq \ldots \leq \gamma_{n} \leq \ldots \leq \gamma$ and

(ii) $\lim _{n \rightarrow \infty} \gamma_{n}(f, f)=\gamma(f, f), f \in \mathscr{\mathscr { D }}$. 
The notion of a regularizing sequence was introduced by Nenciu [8].

Example 2.3. A regularizing sequence of $\gamma$ with respect to $\mathscr{D}$ of Example 2.1 can be defined by

$$
\gamma_{\alpha, n}(f, g)=\frac{1}{4} \int_{\mathbb{R}^{1} \backslash\left[-\frac{1}{n}, \frac{1}{n}\right]} \frac{1}{|x|^{\alpha}} f(x) \overline{g(x)} d x,
$$

$f, g \in \operatorname{dom}\left(\gamma_{\alpha, n}\right)=\mathfrak{h}, 0 \leq \alpha \leq 2$.

Lemma 2.4. Let $\gamma$ be a symmetric form obeying (2.3). If $\left\{\gamma_{n}\right\}_{n=1}^{\infty}$ is a regularizing sequence of $\gamma$ with respect to $\mathscr{D}$, then $\left\{\gamma_{n}\right\}_{n=1}^{\infty}$ is a regularizing sequence of $\hat{\gamma}$ with respect to $\operatorname{dom}(\hat{\gamma})=\operatorname{dom}(\hat{\nu})$.

Proof. By (2.3) and (i) we find the estimate

$$
\gamma_{n}(f, f) \leq \hat{\gamma}(f, f) \leq c\left(\nu(f, f)+\|f\|^{2}\right), \quad f \in \operatorname{dom}(\hat{\nu}),
$$

$c=\max \{a, b\}$, that verifies $0 \leq \gamma_{1} \leq \gamma_{2} \leq \ldots \leq \gamma_{n} \leq \hat{\gamma}$. Hence, the limit $\lim _{n \rightarrow \infty} \gamma_{n}(f, f)$ exists for $f \in \operatorname{dom}(\hat{\gamma})$ and obeys the estimate $\lim _{n \rightarrow \infty} \gamma_{n}(f, f) \leq \hat{\gamma}(f, f)$. By (ii) we have $\lim _{n \rightarrow \infty} \gamma_{n}(f, f)=\hat{\gamma}(f, f)$ if $f \in \mathscr{D}$. If $f \in \operatorname{dom}(\hat{\gamma})=\operatorname{dom}(\hat{\nu})$, then for any $\varepsilon>0$ there is $g \in \mathscr{D}$ such that $c\left(\nu(f-g, f-g)+\|f-g\|^{2}\right)<\varepsilon^{2}$. On account of (2.11) this yields that $\gamma_{n}(f-g, f-g)<\varepsilon^{2}$ uniformly in $n=1,2, \ldots$. Applying the Schwarz inequality and standard $\varepsilon-\delta$ arguments we complete the proof.

If $\left\{\gamma_{n}\right\}_{n=1}^{\infty}$ is a regularizing sequence of $\gamma$ with respect to $\mathscr{D}$, then we can define a perturbed family of closed symmetric forms $\left\{\hat{\mu}_{\kappa, n}\right\}_{n=1}^{\infty}$ by

$$
\hat{\mu}_{\kappa, n}(f, g)=\hat{\nu}(f, g)-\frac{1}{\kappa} \gamma_{n}(f, g), \quad f, g \in \operatorname{dom}\left(\hat{\mu}_{n}\right)=\operatorname{dom}(\hat{\nu}),
$$

$\kappa>a$. On account of (i) the family $\left\{\hat{\mu}_{\kappa, n}\right\}_{n=1}^{\infty}$ is nonincreasing and bounded from below by $\hat{\mu}_{\kappa}$. Moreover, since $\hat{\mu}_{\kappa} \geq \hat{\lambda}_{\kappa}$ we have $\hat{\mu}_{\kappa, n} \geq \hat{\lambda}_{\kappa}, n=1,2, \ldots$ Denoting by $\left\{\hat{H}_{\kappa, n}\right\}$ the associated family of self-adjoint operators we obviously get $\hat{H}_{\kappa, n} \geq \hat{H}_{\kappa} \geq \hat{\lambda}_{\kappa} I, \kappa>a$.

Proposition 2.5. Let $\gamma$ be a symmetric form obeying (2.3). If $\left\{\gamma_{n}\right\}_{n=1}^{\infty}$ is a regularizing sequence of $\gamma$ with respect to $\mathscr{D}$, then the family $\left\{\hat{H}_{\kappa, n}\right\}_{n=1}^{\infty}$ of self-adjoint operators associated with $\left\{\hat{\mu}_{\kappa, n}\right\}_{n=1}^{\infty}$ converges in the strong resolvent sense to $\hat{H}_{\kappa}$ for $\kappa>$ a as $n \rightarrow \infty$, i.e.

$$
\text { s- } \lim _{n \rightarrow \infty}\left(\hat{H}_{\kappa, n}-z\right)^{-1}=\left(\hat{H}_{\kappa}-z\right)^{-1}, \quad z \in \mathbb{C}^{1} \backslash\left[\hat{\lambda}_{\kappa}, \infty\right), \quad a<\kappa
$$

Proof. By Lemma 2.4 we have $\lim _{n \rightarrow \infty} \gamma_{n}(f, f)=\hat{\gamma}(f, f), f \in \operatorname{dom}(\hat{\nu})$, that yields $\lim _{n \rightarrow \infty} \hat{\mu}_{\kappa, n}(f, f)=\hat{\mu}_{\kappa}(f, f),{ }_{\infty}^{n \rightarrow \infty} \in \operatorname{dom}\left(\hat{\mu}_{\kappa}\right)$. Since $\operatorname{dom}\left(\hat{\mu}_{\kappa, n}\right)=\operatorname{dom}(\hat{\nu})$, $n=1,2, \ldots$, we get $\operatorname{dom}\left(\hat{\mu}_{\kappa}\right)=\bigcup_{n=1}^{\infty} \operatorname{dom}\left(\mu_{\kappa, n}\right)$. On the other hand, $\hat{\mu}_{\kappa}$ defined on $\operatorname{dom}\left(\hat{\mu}_{\kappa}\right)$ is a closed symmetric form. Hence, by Theorem 3.11 of $[6$, VIII] we immediately obtain (2.13). 
If $\left\{\gamma_{n}\right\}_{n=1}^{\infty}$ is a regularizing sequence of $\gamma$ with respect to $\mathscr{D}$, then besides (2.12) it is possible to introduce also a family $\left\{\mu_{\kappa, n}\right\}_{n=1}^{\infty}$ of closed symmetric forms defined by

$$
\mu_{\kappa, n}(f, g)=\nu(f, g)-\frac{1}{\kappa} \gamma_{n}(f, g), \quad f, g \in \operatorname{dom}\left(\mu_{\kappa, n}\right)=\operatorname{dom}(\nu)
$$

$\kappa>a$. On account of (i) the sequence $\left\{\mu_{\kappa, n}\right\}_{n=1}^{\infty}$ is nonincreasing too and, in addition

$$
\mu_{\kappa, n} \leq \hat{\mu}_{\kappa, n}, \quad n=1,2, \ldots, \kappa>a .
$$

Let $\left\{H_{\kappa, n}\right\}_{n=1}^{\infty}$ be the family of associated self-adjoint operators. Then we obviously get $H_{\kappa, n} \leq \hat{H}_{\kappa, n}, n=1,2, \ldots$ However, in general, we cannot expect that the forms $\left\{\mu_{\kappa, n}\right\}_{n=1}^{\infty}$ and, therefore, the operators $\left\{H_{\kappa, n}\right\}_{n=1}^{\infty}$ are bounded from below and, moreover, that $\left\{H_{\kappa, n}\right\}_{n=1}^{\infty}$ converges in the strong resolvent sense to $\hat{H}_{\kappa}$. However, this is the contents of the above-described (see Introduction) phenomenon in quantum mechanics. Consequently, to analyse this phenomenon in the frame of our approach we have to restrict a bit the notion of regularizing sequence.

Definition 2.6. Let $\left\{\gamma_{n}\right\}_{n=1}^{\infty}$ be a regularizing sequence of $\gamma$ with respect to $\mathscr{D} \subseteq \operatorname{dom}(\nu)$. The sequence $\left\{\gamma_{n}\right\}_{n=1}^{\infty}$ is called $\hat{\nu}$-admissible if for any nontrivial $f \in \mathscr{M b}=\{h \in \operatorname{dom}(\nu): \nu(h, k)+(h, k)=0, \forall k \in \operatorname{dom}(\hat{\nu})\}$, we have

$$
\sup _{n} \gamma_{n}(f, f)=+\infty \text {. }
$$

To clarify this condition we note that the set $\operatorname{dom}(\nu)$ can be transformed into a Hilbert space $\mathfrak{h}_{\nu}$ by equipping $\operatorname{dom}(\nu)$ with the scalar product $(\cdot, \cdot)_{\nu}$,

$$
(f, g)_{\nu}=\nu(f, g)+(f, g), \quad f, g \in \operatorname{dom}(\nu) .
$$

The set $\operatorname{dom}(\hat{\nu})$ is obviously a closed subspace $\mathfrak{h}_{\hat{\nu}}$ of $\mathfrak{h}_{\nu}$. Moreover, we have $\mathscr{M}=\mathfrak{h}_{\nu} \ominus_{\nu} \hat{\mathfrak{h}}_{\nu}$. Notice that on account of Lemma 2.4 for $k \in \operatorname{dom}(\hat{\nu})$ we always have

$$
\sup _{n} \gamma_{n}(k, k)=\hat{\gamma}(k, k)<+\infty \text {. }
$$

Lemma 2.7. Let $\gamma$ be a symmetric form obeying (2.3) and let $\left\{\gamma_{n}\right\}_{n=1}^{\infty}$ be a regularizing sequence of $\gamma$ with respect to $\mathscr{D}$. Further, let $\mathscr{H}_{\lambda}=\{h \in \operatorname{dom}(\nu): \nu(h, k)-$ $\lambda(h, k)=0, \forall k \in \operatorname{dom}(\hat{\nu})\}, \lambda<0$. If $\left\{\gamma_{n}\right\}_{n=1}^{\infty}$ is $\hat{\nu}$-admissible, then for any nontrivial $f \in \mathscr{M}_{\lambda}, \lambda<0$, the condition (2.16) is also satisfied.

Proof. Obviously, $\mathscr{C}_{\lambda}$ is a closed subspace of $\mathfrak{h}_{\nu}$. Therefore, there are elements $h \in \mathfrak{h}_{\nu} \ominus_{\nu} \hat{\mathfrak{h}}_{\nu}$ and $k \in \hat{\mathfrak{h}}_{\nu}$ such that $f=h+k, f \in \mathscr{M}_{\lambda}$. Assuming $h=0$ we get $f \in \operatorname{dom}(\hat{\nu})$ and $f=0$. Since sup $\gamma_{n}(h, h)=+\infty$ by definition, we obtain (2.16) taking (2.18) into account.

Example 2.8. Let us show that the regularzing sequence $\left\{\gamma_{n}\right\}_{n=1}^{\infty}$ defined in Example 2.3 is $\hat{\nu}$-admissible. By a simple calculation one gets that $\mathscr{l} b=\left\{z e^{-|x|}: z \in \mathbb{C}^{1}\right\}$ and

$$
\gamma_{\alpha, n}\left(e^{-|x|}, e^{-|x|}\right)=\frac{1}{4} \int_{\mathbb{R}^{1} \backslash\left[-\frac{1}{n}, \frac{1}{n}\right]} \frac{1}{|x|^{\alpha}} e^{-2|x|} d x \rightarrow+\infty
$$

as $n \rightarrow \infty$ and $1 \leq \alpha \leq 2$. 
Since $\left\{\gamma_{n}\right\}_{n=1}^{\infty}$ is a family of bounded non-negative symmetric forms on $\mathfrak{h}$, a family of bounded non-negative self-adjoint operators $\left\{V_{n}\right\}_{n=1}^{\infty}$ corresponds to it. Using this family, the self-adjoint operators $\hat{H}_{n}$ and $H_{n}$ can be represented by

$$
\hat{H}_{\kappa, n}=\hat{A}-\frac{1}{\kappa} V_{n}, \quad \mathscr{D}\left(\hat{H}_{\kappa, n}\right)=\mathscr{D}(\hat{A}), \quad n=1,2, \ldots,
$$

and

$$
H_{\kappa, n}=A-\frac{1}{\kappa} V_{n}, \quad \mathscr{D}\left(H_{\kappa, n}\right)=\mathscr{D}(A), \quad n=1,2, \ldots,
$$

$\kappa>a$. Let us introduce the families of bounded non-negative self-adjoint operators $\hat{T}_{\lambda, n}=(\hat{A}-\lambda)^{-1 / 2} V_{n}(\hat{A}-\lambda)^{-1 / 2}$ and $T_{\lambda, n}=(A-\lambda)^{-1 / 2} V_{n}(A-\lambda)^{-1 / 2}$, $n=1,2, \ldots, \lambda<0$.

Lemma 2.9. There is $a \lambda<0$ and an open set $\mathscr{C} \subset(a,+\infty)$ such that $\mathscr{\odot} \subset \varrho\left(T_{\lambda, n}\right)$ for every $n=1,2, \ldots$ if and only if $\lambda \in \varrho\left(H_{\kappa, n}\right)$ for any $\kappa \in$ (Q) and every $n=1,2, \ldots$

Proof. The operator $H_{\kappa, n}-\lambda$ obviously admits the representation

$$
\left(H_{\kappa, n}-\lambda\right) f=\frac{1}{\kappa} \sqrt{A-\lambda}\left(\kappa-T_{\lambda, n}\right) \sqrt{A-\lambda} f, \quad f \in \mathscr{D}(A)
$$

$n=1,2, \ldots, \lambda<0$.

If $\mathcal{G}^{\prime} \subset \varrho\left(T_{\lambda, n}\right)$ for every $n=1,2, \ldots$, then the representation (2.22) yields $\lambda \in \varrho\left(H_{\kappa, n}\right)$ for $\kappa \in \mathcal{O}$ and every $n=1,2, \ldots$

Assume now that $\lambda \in \varrho\left(H_{\kappa, n}\right)$ for any $\kappa \in \mathcal{O}$ and every $n=1,2, \ldots$. Since $\frac{1}{\kappa} V_{n}$ are bounded operators for every $n=1,2, \ldots$, there are $\lambda_{n}<\lambda-\frac{1}{\kappa}\left\|V_{n}\right\|$ such that

$$
H_{\kappa, n}-\lambda_{n}=A-\frac{1}{\kappa} V_{n}-\lambda_{n} \geq I
$$

for $\kappa \in \mathcal{O}$. Furthermore, the identity

$$
\begin{aligned}
& ((A-\lambda) f, f)=\left(\left(H_{\kappa, n}-\lambda_{n}\right) f, f\right)+\left(\left(\frac{1}{\kappa} V_{n}+\lambda_{n}-\lambda\right) f, f\right) \\
& \quad \times\left(\left(H_{\kappa, n}-\lambda_{n}\right) f, f\right), \quad f \in \operatorname{dom}(A)=\operatorname{dom}\left(H_{\kappa, n}\right), \quad \kappa \in \mathscr{Q},
\end{aligned}
$$

yields that $(A-\lambda)^{1 / 2}\left(H_{\kappa, n}-\lambda_{n}\right)^{-1 / 2}$ is a bounded operator for every $n=1,2, \ldots$, and $\kappa \in \mathscr{Q}$. Consequently, the operator

$$
\begin{aligned}
& \sqrt{A-\lambda}\left(H_{\kappa, n}-\lambda\right)^{-1} \sqrt{A-\lambda} \\
& =\sqrt{A-\lambda} \frac{1}{\sqrt{H_{\kappa, n}-\lambda_{n}}} \frac{H_{\kappa, n}-\lambda_{n}}{H_{\kappa, n}-\lambda} \frac{1}{\sqrt{H_{\kappa, n}-\lambda_{n}}} \sqrt{A-\lambda}
\end{aligned}
$$

is well-defined and bounded for any $\kappa \in \mathcal{Q}$ and $n=1,2, \ldots$. Taking the representation (2.22) into account we find

$$
\left(\kappa-T_{\lambda, n}\right)^{-1}=\frac{1}{\kappa} \sqrt{A-\lambda}\left(H_{\kappa, n}-\lambda\right)^{-1} \sqrt{A-\lambda}
$$


which shows that $\left(\kappa-T_{\lambda, n}\right)^{-1}$ is a bounded operator for $\kappa \in \mathcal{O}$ and $N=1,2, \ldots$. Thus, we proved that $\odot \subset \varrho\left(T_{\lambda, n}\right)$ for $n=1,2, \ldots$

In the sequel we need a certain modification of the Theorem 3.6 of [6, VIII].

Lemma 2.10. Let $\left\{T_{n}\right\}_{n=1}^{\infty}$ be a nondecreasing sequence of bounded nonnegative self-adjoint operator defined on some subspace $\mathfrak{k} \subset \mathfrak{h}$. If

$$
\lim _{n \rightarrow \infty}\left(T_{n} k, k\right)=(T k, k), \quad k \in \mathfrak{k}
$$

and

$$
\sup _{n}\left(T_{n} h, h\right)=+\infty, \quad 0 \neq h \in \mathfrak{h} \ominus \mathfrak{k},
$$

then for any open set $\odot \subset \mathbb{C}^{1}$ obeying $\odot \subset \varrho\left(T_{n}\right), n=1,2, \ldots$, and $\odot \subset \varrho(T)$ one gets formula:

$$
\text { s- } \lim _{n \rightarrow \infty}\left(T_{n}-z\right)^{-1}=(T-z)^{-1} \oplus 0, \quad z \in \mathscr{O} \text {. }
$$

Proof. Since $\left\{T_{n}\right\}_{n=1}^{\infty}$ is nondecreasing, the sequence $\left\{\left(T_{n}+I\right)^{-1}\right\}_{n=1}^{\infty}$ is nonincreasing. Therefore, on account of Theorem 3.3 of $[6, \mathrm{VIII}]$ the limit s- $\lim _{n \rightarrow \infty}\left(T_{n}+I\right)^{-1}=X$ exists. Obviously, we have

$$
\left(T_{n}+I\right)^{-1} \geq X, \quad n=1,2, \ldots
$$

Then there is a sequence of contractions $\left\{\Gamma_{n}\right\}_{n=1}^{\infty}$ such that

$$
\sqrt{X}=\Gamma_{n}\left(T_{n}+I\right)^{-1 / 2}, \quad n=1,2, \ldots
$$

Let $f \in \mathfrak{h}$. We have

$$
\begin{aligned}
\sup _{n}\left(T_{n} \sqrt{X} f, \sqrt{X} f\right) & =\sup _{n}\left(T_{n}\left(T_{n}+I\right)^{-1 / 2} \Gamma_{n}^{*} f,\left(T_{n}+I\right)^{-1 / 2} \Gamma_{n}^{*} f\right) \\
& \leq \sup _{n}\left\|\Gamma_{n}^{*} f\right\|^{2} \leq\|f\|^{2} .
\end{aligned}
$$

Taking into account the decomposition $\sqrt{X} f=k+h, k \in \mathfrak{k}, h \in \mathfrak{h} \ominus \mathfrak{k}$, we find

$$
\left\|\sqrt{T_{n}} \sqrt{X} f\right\| \geq\left\|\sqrt{T_{n}} h\right\|-\left\|\sqrt{T_{n}} k\right\|
$$

Therefore, by (2.27) and (2.28) the $\sup _{n}\left\|\sqrt{T_{n}} \sqrt{X} f\right\|=+\infty$ if $h \neq 0$. This contradicts to (2.32). Hence, $h=0$. Consequently, we get $\mathscr{R}(\sqrt{X}) \subseteq \mathfrak{k}$ or, equivalently, $\operatorname{ker}(\sqrt{X}) \supseteq \mathfrak{h} \ominus \mathfrak{k}$. Let us show that $\operatorname{ker}(\sqrt{X})=\mathfrak{h} \ominus \mathfrak{k}$. To this end, we have to show that $k \in \mathfrak{k}$ and

$$
\lim _{n \rightarrow \infty}\left(T_{n}+I\right)^{-1} k=0
$$


yields $k=0$. Since $\varepsilon T_{n}+I \geq \varepsilon T_{n}+\varepsilon I, 0<\varepsilon \leq 1, n=1,2, \ldots$, we find

$$
\lim _{n \rightarrow \infty}\left(\left(\varepsilon T_{n}+I\right)^{-1} k, k\right)=0, \quad 0<\varepsilon \leq 1
$$

By the inequality $\left(\varepsilon T_{n}+I\right)^{-1} \geq I-\varepsilon T_{n}$ we obtain

$$
\varepsilon\left(T_{n} k, k\right) \geq(k, k)-\left(\left(\varepsilon T_{n}+I\right)^{-1} k, k\right), \quad 0<\varepsilon \leq 1
$$

$n=1,2, \ldots$, or

$$
\sup _{n}\left(T_{n} k, k\right) \geq \frac{1}{\varepsilon}(k, k), \quad 0<\varepsilon \leq 1 .
$$

Since $\varepsilon$ can be chosen arbitrary small, $k \neq 0$ implies, in contradiction to (2.27), that $\sup \left(T_{n} k, k\right)=+\infty$. Hence, $k=0$ and $\operatorname{ker}(\sqrt{X})=\mathfrak{h} \ominus \mathfrak{k}$.

Let $P$ be the orthogonal projection from $\mathfrak{h}$ onto $\mathfrak{k}=(\mathscr{R}(\sqrt{X}))^{-}$. Since s$\lim _{n \rightarrow \infty}\left(T_{n}+I\right)^{-1}=X$ implies s- $\lim _{n \rightarrow \infty}\left(T_{n}+I\right)^{-1 / 2}=\sqrt{X}$, from (2.31) we immediately get

$$
\text { s- } \lim _{n \rightarrow \infty} \Gamma_{n} P=P
$$

which yields

$$
\text { w- } \lim _{n \rightarrow \infty} P \Gamma_{n}^{*}=P \text {. }
$$

Moreover, using $P \Gamma_{n}=\Gamma_{n}, n=1,2, \ldots$, by a simple calculation one gets that

$$
\text { s- } \lim _{n \rightarrow \infty} P \Gamma_{n}^{*}=P
$$

Using the representation $\sqrt{T_{n}+I} \sqrt{X}=\Gamma_{n}^{*}, n=1,2, \ldots$, we find

$$
\begin{aligned}
\left\|P \Gamma_{n}^{*} f\right\|^{2} & =\left\|P \sqrt{T_{n}+I} \sqrt{X} f\right\|^{2} \\
& \leq\left(\sqrt{X}\left(T_{n}+I\right) \sqrt{X} f, f\right) \leq\|f\|^{2}, \quad f \in \mathfrak{h}, \quad n=1,2, \ldots
\end{aligned}
$$

Let $n \rightarrow \infty$ in (2.41) and using (2.27) and (2.40) we get

$$
\|P f\|^{2}=(\sqrt{X}(T+I) \sqrt{X} f, f), \quad f \in \mathfrak{h} .
$$

Therefore, choosing $f \in \mathfrak{k}$ we obtain $P=\sqrt{X}(T+I) \sqrt{X}$. Moreover, $(\mathscr{R}(\sqrt{X}))^{-}=\mathfrak{k}$ immediately yields $X=(T+I)^{-1} \oplus 0$ which shows (2.29) for $z=-1$. Now by standard arguments we can extend the proof to a neighbourhood of $z=-1$ and from this neighbourhood to an arbitrary open set $\mathscr{Q}$ obeying the assumptions of the theorem.

Let $\mathscr{L}_{\infty}($.$) be the set of compact operators on a Hilbert space. Then the previous$ lemma can be strengthened as follows:

Corollary 2.11. If in addition to the assumptions of Lemma 2.10 the conditions $T \in \mathscr{L}_{\infty}(\mathfrak{k})$ and $\operatorname{dim}(\mathfrak{h} \ominus \mathfrak{k})<+\infty$ are satisfied, then the strong convergence in (2.29) can be replaced by the operator norm convergence. 
Proof. Since $\left\{\left(T_{n}+I\right)^{-1}\right\}_{n=1}^{\infty}$ is nonincreasing, we get $T(T+I)^{-1} \oplus I_{\mathfrak{h} \ominus \mathfrak{k}} \geq$ $T_{n}\left(T_{n}+I\right)^{-1}, n=1,2, \ldots$, where $I_{\mathfrak{h} \ominus \mathfrak{k}}$ is the identity on $\mathfrak{h} \ominus \mathfrak{k}$. By $T \in \mathscr{L}_{\infty}(\mathfrak{k})$ and $\operatorname{dim}(\mathfrak{h} \ominus \mathfrak{k})<+\infty$ we find $T_{n}\left(T_{n}+I\right)^{-1} \in \mathscr{L}_{\infty}(\mathfrak{h}), n=1,2, \ldots$ Using the representation

$$
\left(T_{n}+I\right)^{-1}-\left\{(T+I)^{-1} \oplus 0\right\}=-T_{n}\left(T_{n}+I\right)^{-1}+\left\{T(T+I)^{-1} \oplus I_{\mathfrak{h} \ominus \mathfrak{k}}\right\},
$$

we obtain

$$
\left(T_{n}+I\right)^{-1}-\left\{(T+I)^{-1} \oplus 0\right\} \in \mathscr{L}_{\infty}(\mathfrak{h}), \quad n=1,2, \ldots
$$

Then applying Theorem 3.5 of [6, VIII] one finds

$$
\|\cdot\|-\lim _{n \rightarrow \infty}\left(T_{n}+I\right)^{-1}=(T+I)^{-1} \oplus 0 .
$$

To prove (2.29) for $z \in \mathscr{O}$ it is enough to repeat the previous line of reasoning, replacing the strong resolvent convergence by the operator norm one.

Theorem 2.12. Let $\gamma$ be a symmetric form obeying (2.3) and let $\left\{\gamma_{n}\right\}_{n=1}^{\infty}$ be a regularizing sequence of $\gamma$ with respect to $\mathscr{D}$. If $\left\{\gamma_{n}\right\}_{n=1}^{\infty}$ is $\hat{\nu}$-admissible and if there is an open set $\mathscr{Q} \subset(a, \infty)$ and $a \lambda<0$ such that $\lambda<\hat{\lambda}_{\kappa}$ and $\lambda \in \varrho\left(H_{\kappa, n}\right)$ for any $\kappa \in \mathcal{O}$ and $n=1,2, \ldots$, then $\left\{H_{\kappa, n}\right\}_{n=1}^{\infty}$ converges in the strong resolvent sense to $\hat{H}_{\kappa}$ for every $\kappa \in \odot$ as $n \rightarrow \infty$, i.e.

$$
\text { s- } \lim _{n \rightarrow \infty}\left(H_{\kappa, n}-z\right)^{-1}=\left(\hat{H}_{\kappa}-z\right)^{-1}, \quad z \in\{\lambda\} \cup \mathbb{C}^{1} \backslash \mathbb{R}^{1}, \quad \kappa \in \mathscr{O} .
$$

The strong convergence can be replaced by the operator norm convergence if the symmetric form $\gamma$ in addition is relatively compact with respect to the symmetric form $\hat{\nu}$ and $\operatorname{dim}(\mathscr{C})<+\infty$.

Proof. Since $\nu, \hat{\nu} \geq 0$, we have $A \geq 0$ and $\hat{A} \geq 0$. Taking into account (2.2) we get the existence of an isometry $W, W^{*} W=I$, such that

$$
\sqrt{A-\lambda} f=W \sqrt{\hat{A}-\lambda} f, \quad f \in \operatorname{dom}(\hat{\nu}) \subseteq \operatorname{dom}(\nu)
$$

which yields

$$
(\hat{A}-\lambda)^{-1 / 2} f=(A-\lambda)^{-1 / 2} W f, \quad f \in \mathfrak{h} .
$$

The symmetric form

$$
\hat{\beta}(f, g)=\hat{\gamma}\left((\hat{A}-\lambda)^{-1 / 2} f,(\hat{A}-\lambda)^{-1 / 2} g\right), \quad f, g \in \mathfrak{h}
$$

is, obviously, well-defined and, moreover, bounded by (2.5). By $\hat{T}_{\lambda}$ we denote the bounded non-negative self-adjoint operator associated with $\hat{\beta}$. Then by Lemma 2.4 we get

$$
\lim _{n \rightarrow \infty} \gamma_{n}\left((\hat{A}-\lambda)^{-1 / 2} f,(\hat{A}-\lambda)^{-1 / 2} f\right)=\hat{\beta}(f, f), \quad f \in \mathfrak{h}
$$

which yields

$$
\text { s- } \lim _{n \rightarrow \infty} \hat{T}_{\lambda, n}=\hat{T}_{\lambda} \text {. }
$$


Let us now calculate the limit

$$
\lim _{n \rightarrow \infty} \gamma_{n}\left((A-\lambda)^{-1 / 2} g,(A-\lambda)^{-1 / 2} g\right), \quad g \in \mathfrak{h}
$$

On account of (2.48) and (2.50) for any $f \in P \mathfrak{h}, P=W W^{*}$, one gets

$$
\begin{aligned}
& \lim _{n \rightarrow \infty} \gamma_{n}\left((A-\lambda)^{-1 / 2} \operatorname{Pf},(A-\lambda)^{-1 / 2} P f\right) \\
& \quad=\lim _{n \rightarrow \infty} \gamma_{n}\left((\hat{A}-\lambda)^{-1 / 2} W^{*} f,(\hat{A}-\lambda)^{-1 / 2} W^{*} f\right) \\
& \quad=\hat{\beta}\left(W^{*} f, W^{*} f\right)=\left(W \hat{T}_{\lambda} W^{*} f, f\right) .
\end{aligned}
$$

If $g \perp \mathscr{R}(W)$, then by (2.48) we have

$$
\begin{aligned}
& \nu\left((A-\lambda)^{-1 / 2} g,(\hat{A}-\lambda)^{-1 / 2} f\right)-\lambda\left((A-\lambda)^{-1 / 2} g,(\hat{A}-\lambda)^{-1 / 2} f\right) \\
& \quad=\nu\left((A-\lambda)^{-1 / 2} g,(A-\lambda)^{-1 / 2} W f\right)-\lambda\left((A-\lambda)^{-1 / 2} g,(A-\lambda)^{-1 / 2} W f\right) \\
& \quad=(g, W f)=0, \quad f \in \mathfrak{h} .
\end{aligned}
$$

Hence $g \perp \mathscr{R}(W)$ yields $(A-\lambda)^{-1 / 2} g \in \mathscr{M}_{\lambda}$. Applying Lemma 2.7 we obtain

$$
\sup _{n} \gamma_{n}\left((A-\lambda)^{-1 / 2} g,(A-\lambda)^{-1 / 2} g\right)=+\infty
$$

if $g \perp \mathscr{R}(W), g \neq 0$. But (2.52) and (2.54) show that the assumptions of Lemma 2.10 are satisfied for $\left\{T_{\lambda, n}\right\}_{n=1}^{\infty}$ and $\mathfrak{k}=\mathscr{B}(W)$. Therefore, applying Lemma 2.9 and Lemma 2.10 we get

$$
\text { s- } \lim _{n \rightarrow \infty}\left(T_{\lambda, n}-\kappa\right)^{-1}=\left(W \hat{T}_{\lambda} W^{*}-\kappa\right)^{-1} \oplus 0
$$

(with respect to the decomposition $\mathfrak{h}=\mathscr{R}(W) \oplus \mathscr{R}(W)^{\perp}$ ) or, equivalently,

$$
\text { s- } \lim _{n \rightarrow \infty}\left(T_{\lambda, n}-\kappa\right)^{-1}=W\left(\hat{T}_{\lambda}-\kappa\right)^{-1} W^{*} \text {. }
$$

Notice that $\kappa \in \varrho\left(\hat{T}_{\lambda}\right)$ is guaranteed by the assumption $\lambda<\hat{\lambda}_{\kappa}, \kappa \in \mathscr{Q}$. Multiplying the right- and left-hand sides of (2.56) by $(A-\lambda)^{-1 / 2}$ and taking into account (2.48) we obtain

$$
\text { s- } \begin{gathered}
\lim _{n \rightarrow \infty}(A-\lambda)^{-1 / 2}\left(T_{\lambda, n}-\kappa\right)^{-1}(A-\lambda)^{-1 / 2} \\
=(\hat{A}-\lambda)^{-1 / 2}\left(\hat{T}_{\lambda}-\kappa\right)^{-1}(\hat{A}-\lambda)^{-1 / 2}
\end{gathered}
$$

Now taking into account (2.26) we get

$$
\text { s- } \lim _{n \rightarrow \infty}\left(H_{\kappa, n}-\lambda\right)^{-1}=\left(\hat{H}_{\kappa}-\lambda\right)^{-1}
$$

which proves (2.46) for $z=\lambda$. Using Theorem 1.2 and Corollary 1.4 of [6, VIII] we complete the proof of the first part of the theorem.

To prove the second part we note that the relative compactness of $\hat{\gamma}$ with respect to $\hat{\nu}$ yields $\hat{T}_{\lambda} \in \mathscr{C}_{\infty}(\mathfrak{h})$. Furthermore, by stability arguments (cf. Lemma 2.7 ) one gets $\operatorname{dim}(\mathfrak{h} \ominus \mathfrak{k})=\operatorname{dim}\left(\mathscr{C}_{\lambda}\right)=\operatorname{dim}(\mathscr{C})<+\infty$. Applying Corollary 2.11 we find that 
the strong convergence in (2.55) can be replaced by the operator norm convergence. With this the remaining part is obvious.

To apply these results to our example we have to verify the existence of a real

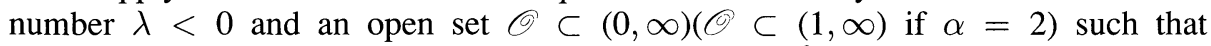
$\lambda \in \varrho\left(H_{\kappa, n}\right)$ and for $n=1,2, \ldots, \kappa \in \Theta . H_{\kappa, n}=-\frac{d^{2}}{d x^{2}}-\frac{1}{4 \kappa} \frac{1}{|x|^{\alpha}} \chi_{\mathbb{R}^{1} \backslash\left[-\frac{1}{n}, \frac{1}{n}\right]}(x)$.

In general, it is not easy to show this and needs additional efforts. However, the situation becomes simpler if the operators $A$ and $\hat{A}$ satisfy additional properties. In the following section such additional conditions are introduced and investigated.

\section{Special Case}

In the following we make the additional assumption $m=\operatorname{codim}(\operatorname{dom}(\hat{\nu}))=$ $\operatorname{dim}(\operatorname{dom}(\nu) / \operatorname{dom}(\hat{\nu}))<+\infty$. Under this additional condition Theorem 2.12 can be strengthened as follows.

Theorem 3.1. Let $\gamma$ be a symmetric form obeying (2.3) and let $m=\operatorname{codim}(\operatorname{dom}(\hat{\nu}))<$ $+\infty$. If $\left\{\gamma_{n}\right\}_{n=1}^{\infty}$ is a $\hat{\nu}$-admissible regularizing sequence of $\gamma$, then for any $\kappa \in(a, \infty)$, except a finite set of at most $m$ points, there is a $\lambda<\hat{\lambda}_{\kappa}$ such that $\lambda \in \varrho\left(H_{\kappa, n}\right)$ for sufficiently large $n$ and $\mathrm{s}-\lim _{n \rightarrow \infty}\left(H_{\kappa, n}-z\right)^{-1}=\left(\hat{H}_{\kappa}-z\right)^{-1}$ for $z \in\{\lambda\} \cup \mathbb{C}^{1} \backslash \mathbb{R}^{1}$.

The strong convergence can be replaced by the operator norm convergence if the symmetric form $\gamma$ is in addition relatively compact with respect to the symmetric form $\hat{\nu}$.

Proof. Let $\hat{\lambda}_{\kappa}=\inf \sigma\left(\hat{H}_{\kappa}\right), \kappa>a$. By the KLMN-Theorem [10, Theorem X.17] we obtain the estimate $\hat{\lambda}_{\kappa} \geq-\frac{b}{a}$. Hence $\hat{\lambda}=\inf _{\kappa>a} \lambda_{\kappa}$ is finite. Since $0 \leq \gamma_{n} \leq \hat{\gamma}$, $n=1,2, \ldots$, one obviously gets

$$
\inf \sigma\left(\hat{H}_{\kappa, n}\right)=\hat{\lambda}_{\kappa, n} \geq \hat{\lambda}_{\kappa} \geq \hat{\lambda}, \quad \kappa>a, \quad n=1,2, \ldots
$$

Moreover, by (2.15) we find

$$
\hat{\lambda}_{\kappa, n} \geq \lambda_{\kappa, n}=\inf \sigma\left(H_{\kappa, n}\right), \quad \kappa>a, \quad n=1,2, \ldots
$$

Since $\operatorname{dom}\left(\mu_{\kappa, n}\right)=\operatorname{dom}(\nu)$ and $\operatorname{dom}\left(\hat{\mu}_{\kappa, n}\right)=\operatorname{dom}(\hat{\nu})$ for $\kappa>a$ and $n=1,2, \ldots$, we get $\operatorname{codim}\left(\operatorname{dom}\left(\hat{\mu}_{\kappa, n}\right)\right)=m$. On account of $\mu_{\kappa, n} \mid \operatorname{dom}\left(\hat{\mu}_{\kappa, n}\right)=\hat{\mu}_{\kappa, n}$, for any $\lambda<\lambda_{\kappa, n}$ there exists an isometry $W_{\kappa, n}$ such that

$$
\sqrt{H_{\kappa, n}-\lambda} f=W_{\kappa, n} \sqrt{\left(\hat{H}_{\kappa, n}-\lambda\right)} f
$$

$f \in \operatorname{dom}\left(\hat{\mu}_{\kappa, n}\right) \subseteq \operatorname{dom}\left(\mu_{\kappa, n}\right)$, which yields

$$
\left(\hat{H}_{\kappa, n}-\lambda\right)^{-1 / 2} f=\left(H_{\kappa, n}-\lambda\right)^{-1 / 2} W_{\kappa, n} f, \quad f \in \mathfrak{h} .
$$

The last relation implies that $m=\operatorname{codim}\left(\operatorname{dom}\left(\hat{\mu}_{\kappa, n}\right)\right)=\operatorname{codim}\left(\mathscr{R}\left(W_{\kappa, n}\right)\right)$ for $\kappa>a$ and $n=1,2, \ldots$. Hence, the range of the projection $Q_{\kappa, n}=I-W_{\kappa, n} W_{\kappa, n}^{*}$ is an $m$-dimensional subspace. 
From (3.4) we obtain the following representation

$$
\begin{aligned}
& \left(H_{\kappa, n}-\lambda\right)^{-1}-\left(\hat{H}_{\kappa, n}-\lambda\right)^{-1} \\
& \quad=\left(H_{\kappa, n}-\lambda\right)^{-1 / 2} Q_{\kappa, n}\left(H_{\kappa, n}-\lambda\right)^{-1 / 2}, \quad \kappa>a, \quad n=1,2, \ldots
\end{aligned}
$$

Therefore, the resolvent difference $\left(H_{\kappa, n}-\lambda\right)^{-1}-\left(\hat{H}_{\kappa, n}-\lambda\right)^{-1}$ is an $m$-dimensional operator for $\kappa>a$ and $n=1,2, \ldots$.

Denoting by $E_{\kappa, n}($.$) the spectral measure of H_{\kappa, n}$ we can introduce the integer function $\pi_{\kappa, n}=\operatorname{dim}\left(E_{\kappa, n}((-\infty, \hat{\lambda}) \mathfrak{h}), \kappa>a, n=1,2, \ldots\right.$. Further, we note that the spectrum of $\hat{H}_{\kappa, n}$ on the interval $(-\infty, \hat{\lambda})$ is empty. Thus, applying Theorem 3 of [2, IX, Sect. 3] we find that $0 \leq \pi_{\kappa, n} \leq m$ holds for any $\kappa>a$ and $n=1,2, \ldots$, i.e., the spectrum of $H_{\kappa, n}$ on the interval $(-\infty, \hat{\lambda})$ consists of at most $m$ eigenvalues counting multiplicities.

Taking into account Lemma 1 of [2, IX, Sect. 4] it is easy to check that

$$
\pi_{\kappa, n} \leq \pi_{\kappa^{\prime}, n^{\prime}}, \quad a<\kappa^{\prime} \leq \kappa, \quad n^{\prime} \geq n \geq 1
$$

Since $0 \leq \pi_{\kappa, n} \leq m$, from (3.6) we get that the limit $\pi_{\kappa}=\lim _{n \rightarrow \infty} \pi_{\kappa, n}$ always exists for $\kappa>a$ and obeys $0 \leq \pi_{\kappa} \leq m$. Moreover, the function $\pi_{\kappa}$ is non-increasing on $(0, \infty)$. Denoting by $\mathscr{N}$ the set of jump-points of $\pi_{\kappa}$ on $(a, \infty)$, one can check that $\mathscr{N}$ consists of at most $m$ points, i.e. $\mathscr{N}=\bigcup_{j=1}^{p}\left\{\kappa_{j}\right\}, 1 \leq p \leq m$, which we assume to be ordered as $\kappa_{p}<\kappa_{p-1}<\ldots<\kappa_{1}<+\infty$. Notice that $\mathscr{N}$ can be empty.

Next we show that for any $\kappa \in(a, \infty) \backslash \mathscr{N}$ the conclusions of the theorem hold. If $\kappa \in(a, \infty) \backslash \mathscr{N}$ we find a $j, 0 \leq j \leq p+1$, and $\alpha \in(a, \infty)$ such that $\kappa_{j+1}<\kappa<\alpha<\kappa_{j}$, where we have set $\kappa_{p+1}=a$ and $\kappa_{0}=+\infty$. Note that on the intervals $\left\{\left(\kappa_{j+1}, \kappa_{j}\right)\right\}_{j=0}^{p+1}$ the function $\pi_{\kappa}$ is constant. Suppose that $\pi_{\kappa} \mid\left(\kappa_{j+1}, \kappa_{j}\right)=\pi_{j}, j=0,1,2, \ldots, p$. Since $\lim _{n \rightarrow \infty} \pi_{\alpha, n}=\pi_{j}$, there is a real number $n_{\alpha}$ such that $n \geq n_{\alpha}$ yields $\pi_{\alpha, n}=\pi_{j}$. Moreover, on account of (3.6) we get $\pi_{\kappa, n}=\pi_{j}$ for every $\kappa \in\left(\kappa_{j+1}, \alpha\right)$ and $n \geq n_{\alpha}$.

Let $\left\{\lambda_{\kappa, n}^{(r)}\right\}_{r=1}^{\pi_{j}}$ be the eigenvalues (counting multiplicities) of $H_{\kappa, n}, \kappa \in\left(\kappa_{j+1}, \alpha\right)$, $n \geq n_{\alpha}$, on $(-\infty, \hat{\lambda})$ ordered by

$$
-\infty<\lambda_{\kappa, n}^{(1)} \leq \lambda_{\kappa, n}^{(2)} \leq \ldots \leq \lambda_{\kappa, n}^{\left(\pi_{\jmath}\right)}<\hat{\lambda}
$$

Then applying again Lemma 1 of [2, IX, Sect. 4] we see that the eignevalues $\left\{\lambda_{\kappa, n}^{(r)}\right\}_{r=1}^{\pi_{j}}$ can shift only to the left when $\kappa$ decreases and $n$ increases, i.e.

$$
\lambda_{\kappa^{\prime}, n^{\prime}}^{(r)} \leq \lambda_{\kappa, n}^{(r)}, \quad r=1,2, \ldots, \pi_{j}
$$

$\kappa_{j+1}<\kappa^{\prime} \leq \kappa<\alpha$ and $n_{\alpha} \leq n \leq n^{\prime}$. Consequently, choosing $\lambda$ from the interval $\left(\lambda_{\alpha, n_{\alpha}}^{\pi_{j}}, \hat{\lambda}\right)$ we obtain that $\lambda \in \varrho\left(H_{\kappa, n}\right)$ for $\kappa \in \mathscr{C} \stackrel{\text { def }}{=}\left(\kappa_{j+1}, \alpha\right)$ and $n \geq n_{\alpha}$. Applying Theorem 2.12 we finish the first part of the theorem.

To prove the second part we equip the set $\operatorname{dom}(\nu)$ with the scalar' product $(., .)_{\nu}$. Then the set $\operatorname{dom}(\nu)$ transforms into the Hilbert space $\mathfrak{h}_{\nu}$ and $\operatorname{dom}(\hat{\nu})$ forms a closed subspace $\hat{\mathfrak{h}}_{\nu}$ in $\mathfrak{h}_{\nu}$. Thus, applying Lemma 1.40 of $[6$, III] we find 
$\operatorname{dim}(\mathscr{l})=m<+\infty, \mathscr{l} b=\mathfrak{h} \ominus_{\nu} \hat{\mathfrak{h}}_{\nu}$. Therefore, taking into account the relative compactness of the symmetric form $\gamma$ with respect to $\hat{\nu}$ and Theorem 2.12 we complete the proof.

Remark 3.2. In view of the geometrical meaning of the deficiency indices, it is clear that the conditon $\operatorname{codim}(\operatorname{dom}(\hat{\nu}))<+\infty$ is satisfied if we require that $\mathscr{D} \subseteq \mathscr{D}(A)$ and the closure $\stackrel{\circ}{A}$ of the operator $A \mid \mathscr{D}$ has finite deficiency indices.

Example 3.3. Proceeding with Example 2.1 and Example 2.8 we see that in this case $\operatorname{dim}(\operatorname{dom}(\nu) / \operatorname{dom}(\hat{\nu}))=1$. Moreover, using Example 2.3 and again Example 2.8 together with Theorem 3.1 we get that $\left\{H_{\kappa, n}\right\}_{n=1}^{\infty}$ given by $H_{\kappa, n}=$ $-\frac{d^{2}}{d x^{2}}-\frac{1}{\kappa} \frac{1}{4} \frac{1}{|x|^{\alpha}} \chi_{\mathbb{R}^{1} \backslash\left[-\frac{1}{n}, \frac{1}{n}\right]}(x), 1 \leq \alpha \leq 2$, converges in the strong resolvent sense, as $n \rightarrow \infty$, to the Friedrichs extension $\hat{H}_{\kappa}$ of $\left(-\frac{d^{2}}{d x^{2}}-\frac{1}{\kappa} \frac{1}{4} \frac{1}{|x|^{\alpha}}\right) \mid C_{0}^{\infty}\left(\mathbb{R}^{1} \backslash\{0\}\right)$ except at most for one value of the coupling constant $\frac{1}{\kappa}, \kappa>0(\kappa>1$ if $\alpha=2)$. However, this exceptional value can be exluced. To this end we note that the perturbations $V_{n}=\frac{1}{4} \frac{1}{|x|^{\alpha}} \chi_{\mathbb{R}^{1} \backslash\left[-\frac{1}{n}, \frac{1}{n}\right]^{(x), 1}} \leq \alpha \leq 2$, are relatively compact with respect to the usual Laplace operator $A=-\frac{d^{2}}{d x^{2}}$. Consequently, the negative spectrum of $H_{\kappa, n}$ consists of discrete eigenvalues (Theorem 5.35 of [6, IV]). Furthermore, the exact lower bounds $\lambda_{\kappa, n}=\inf \sigma\left(H_{\kappa, n}\right)$ tend to minus infinity as $n \rightarrow \infty$ for any $\kappa>0$. This can be proven by simple calculations with the corresponding quadratic forms. Since $\lambda_{\kappa, n} \in \sigma\left(H_{\kappa, n}\right)$, we obtain that $\lambda_{\kappa, n}$ is an eigenvalue for $\kappa>0$ and sufficiently large $n$. This yields that for $\kappa>0$ and sufficiently large $n$ the eigenvalues $\lambda_{\kappa, n}$ are below the bound $\hat{\lambda}=\inf _{\kappa>0(1 \text { if } \alpha=2)} \sigma\left(\hat{H}_{\kappa}\right)$. Hence the integer-valued function $\pi_{\kappa, n}=\operatorname{dim}\left(E_{\kappa, n}(-\infty, \hat{\lambda}) \mathfrak{h}\right), \kappa>0(>1$ if $\alpha=2)$, equals one for sufficiently large $n$. Obviously, this implies that $\pi_{\kappa}=\lim _{n \rightarrow \infty} \pi_{\kappa, n}=1$ for $\kappa>0(>1$ if $\alpha=2)$. Therefore, the function $\pi_{\kappa}$ has no jump-points that excludes the above-mentioned exceptional value of the coupling constant $\frac{1}{\kappa}$. Hence for $\kappa>0(>1$ if $\alpha=2)$ the sequence $\left\{H_{\kappa, n}\right\}_{n=1}^{\infty}$ converges in the strong resolvent sense to $\hat{H}_{\kappa}$ as $n \rightarrow \infty$.

Since for $1 \leq \alpha<2$ the symmetric form $\gamma_{\alpha}$ is relatively compact with respect to the symmetric form $\hat{\nu}$, the strong convergence can be replaced by the norm convergence.

Notice that the symmetric form $\gamma_{\alpha=2}$ is not relatively compact with respect to $\hat{\nu}$. Hence, the question whether the strong convergence can be replaced in this case by the norm convergence remains open.

\section{Concluding Remarks}

From Theorem 2.12 it follows that Nenciu's conditions (ii) and (iii) of the Theorem 1 [8] can be relaxed, namely (cf. Introduction):

1. Condition (ii)(1) can be understood in the form sense.

2. Condition (ii)(2) can be replaced by the monotonicity of the regularizing sequence, i.e. $-V_{n} \geq-V$ and $-V_{n} \rightarrow-V$ as $n \rightarrow+\infty$.

3. The condition (ii)(3) is not necessary in the abstract approach. However, under such assumption foregoing conclusions can be obtained (see Sect. 3). 
4. The condition (iii) is a consequence of the relative compactness of $\gamma$ with respect to the symmetric form $\hat{\nu}$. To this end, we note that by Theorem VIII. 20 of [9] the norm resolvent convergence yields that for every continuous function $\varphi$ tending to zero at infinity we have $\left\|\varphi\left(H_{\kappa, n}\right)-\varphi\left(\hat{H}_{\kappa}\right)\right\| \rightarrow 0, \kappa \in \mathscr{O}$, as $n \rightarrow \infty$. Choosing $\varphi$ with $\operatorname{supp}(\varphi) \subset\left(-\infty, \hat{\lambda}_{\kappa}\right)$ we obviously get $\left\|\varphi\left(H_{\kappa, n}\right)\right\| \rightarrow 0, \kappa \in \mathscr{Q}$, as $n \rightarrow \infty$. Hence, we find $\left\|E_{\kappa, n}((a, b))\right\| \rightarrow 0, \kappa \in \mathscr{O}$, as $n \rightarrow \infty$ for each interval $(a, b)$ with $-\infty<a<b<\hat{\lambda}_{\kappa}$. Therefore, for interval $(a, b)$ obeying $-\infty<a<b<\hat{\lambda}_{\kappa}$ we find an $n_{0}$ such that $n \geq n_{0}$ implies $E_{\kappa, n}(a, b)=0$. Consequently, there are $c_{n}<\hat{\lambda}_{\kappa}$, obeying $\lim _{n \rightarrow \infty} c_{n}=+\infty$, such that the spectrum of $H_{\kappa, n}$ is situated in $\left(-\infty, c_{n}\right) \cup\left(\hat{\lambda}_{\kappa}-\varepsilon,+\infty\right)$ where $\varepsilon>0$ is arbitrary small.

In the paper [8] the importance of $\hat{\nu}$-admissibility of the regularizing sequence had not been clearly pointed out. Here we show that it is this notion that is the key to the understanding of what is going on in [8] as well as to the abstract operator formulation of the removing regularization theory for singular perturbations.

Finally we would like to stress that our conditions in the main Theorems 2.12 and 3.1 are almost necessary in the following sense: The only condition that is "more than optimal" is the requirement that $\lambda \in \varrho\left(H_{\kappa, n}\right)$ must be satisfied uniformly with respect to small variations of the coupling constant $\frac{1}{\kappa}$.

Acknowledgements. One of the authors (V.Z.) is grateful to the Sonderforschungsbereich 288 (TU Berlin) for hospitality and for financial support extended to him during the completion of the paper. Finally, we would like to thank the referee for his useful remarks.

\section{References}

1. Albeverio, S., Gesztesy, F., Hoegh-Krohn, R., Holden, H.: Solvable models in quantum mechanics. Berlin, Heidelberg, New York: Springer 1988

2. Birman, M.S., Solomjak, M.Z.: Spectral theory of self-adjoint operators in the Hilbert space. Leningrad: Izd. Leningrad Univ. 1980 (in Russian)

3. Faris, W.G.: Self-adjoint operators. Lecture Notes in Mathematics, Vol. 433. Berlin, Heidelberg, New York: Springer 1973

4. Gesztesy, F.: On the one-dimensional Coulomb Hamiltonian. J. Phys. A: Math. Gen. 13, 867-875 (1980)

5. Harrell, E.M.: Singular perturbation potentials. Ann. Phys. 105, 379-406 (1977)

6. Kato, T.: Perturbation theory for linear operators. Berlin, Heidelberg, New York: Springer 1966

7. Klaus, M.: Removing cut-offs from one-dimensional Schrödinger operators. J. Phys. A: Math. Gen. 13, L 295-L 298 (1980)

8. Nenciu, G.: Removing cut-offs from singular perturbations: an abstract result. Lett. Math. Phys. 7, 301-306 (1983)

9. Reed, M., Simon, B.: Methods of modern mathematical physics. I. Functional analysis. New York, London: Academic Press 1972

10. Reed, M., Simon, B.: Methods of modern mathematical physics. II. Fourier analysis, Selfadjointness. New York, San Francisco, London: Academic Press 1975

11. Schechter, M.: Cut-off potentials and forms extensions. Lett. Math. Phys. 1, 265-273 (1976)

12. Simon, B.: Quantum Mechanics for Hamiltonians defined as quadratic forms. Princeton, NJ: Princeton University Press 1971

13. Simon, B.: Quadratic forms and Klauder's phenomenon: a remark on very singular perturbations. J. Funct. Anal. 14, 295-298 (1973)

14. Zagrebnov, V.A.: On singular potential interactions in quantum statistical mechanics. Trans. Moscow Math. Soc. 41, 101-120 (1980) 